\title{
Wireless Channel Losses and Emperical Channel Models
}

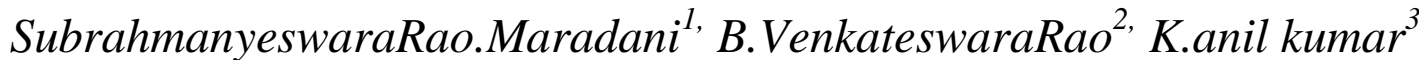 \\ ${ }^{11}$ Dept of ECE, Chalapathi Institute of Engineering and Technology, LAM-522034,Andhra Pradesh ,India) \\ ${ }^{21}$ Dept of ECE, Chalapathi Institute of Engineering and Technology, LAM-522034,Andhra Pradesh ,India) \\ ${ }^{31}$ Dept of ECE, Chalapathi Institute of Engineering and Technology, LAM-522034,Andhra Pradesh ,India)
}

\begin{abstract}
The channel is one of the essential elements of the transmission chain .The wireless channel environment governs the performance of wireless communication systems, since the environment is unpredictable and dynamic.This will make the analysis of the wireless communication system difficult. To that end we classify the wireless channel model. In wireless communications, obstacles, such as houses, buildings, trees and mountains cause reflection, diffraction, scattering and shadowing of the transmitted signals and multipath propagation. Due to the multipath the transmitted signals arrive in different phase angles, amplitude and time interval. The fading is the amplitude fluctuation of the received signal caused by the frequency selective or time variant of the multipath channel. In this paper This paper presents the result for the free space path loss for $1 \mathrm{~km}$ and $5 \mathrm{~km}$ Range of transmitter and receiver are uniform. Also provide the trends between losses and heights of obstacles and antennas The behavior of path losses at various models are discussed and concluded that among the communication models Okumura model shows the least path loss and Cost-231 model shows the largest path loss.
\end{abstract}

Keywords: Channel Models, path loss, height of obstacle, other empirical models

\subsection{Fading Channels}

\section{Introduction}

In wireless communications, obstacles, such as houses, buildings, trees and mountains cause reflection, diffraction, scattering and shadowing of the transmitted signals and multipath propagation. Due to the mutipath the transmitted signals arrive in different phase angles, amplitude and time interval. The fading is the amplitude fluctuation of the received signal caused by the frequency selective or time variant of the multipath channel. The fading process can follow Rayleigh probability distribution or Rician probability distribution, this will depend on the strength of scattering components during transmission The mobile radio channel is usually evaluated from 'statistical' propagation models: no specific terrain data is considered, and channel parameters are modeled as stochastic variables. Three mutually independent, multiplicative propagation phenomena can usually be distinguished: multipath fading, shadowing and 'large-scale' path loss.

Multipath propagation: Fading leads to rapid fluctuations of the phase and amplitude of the signal if the vehicle moves over a distance in the order of a wave length or more. Multipath fading thus has a 'small-scale' effect.

Shadowing: This is a 'medium-scale' effect: field strength variations occur if the antenna is displaced over distances larger than a few tens or hundreds of metres.

The Large scale effects determine a power level averaged over an area of tens or hundreds of metres and therefore called the 'area-mean' power. Shadowing introduces additional fluctuations, so the received local-mean power varies around the area-mean. The term 'local-mean' is used to denote the signal level averaged over a few tens of wave lengths, typically 40 wavelengths. This ensures that the rapid fluctuations of the instantaneous received power due to multipath effects are largely removed.

Path Loss: Path loss models describe the signal attenuation between a transmit and a receive antenna as a function of the propagation distance and other parameters. Some models include many details of the terrain profile to estimate the signal attenuation, whereas others just consider carrier frequency and distance. Antenna heights are other critical parameters

Path loss is one of the mechanisms causing attenuation between the transmitter power amplifier and receiver front end. Some other effects are listed below, with an indication of the order of magnitude in a GSM -like system

- Losses in the antenna feeder $(0 . .4 \mathrm{~dB})$

- Losses in transmit filters, particularly if the antenna radiates signal of multiple transmitters $(0 \ldots 3 \mathrm{~dB})$

- Antenna Directivity gain $(0 . .12 \mathrm{~dB})$

- Losses in duplex filter

- Fade margins to anticipate for multipath $(9 . .19 \mathrm{~dB})$ and shadow losses $(5 \mathrm{~dB})$

- Penetration losses if the receiver is indoors, typically about $10 \mathrm{~dB}$ for $900 \mathrm{MHz}$ signals 


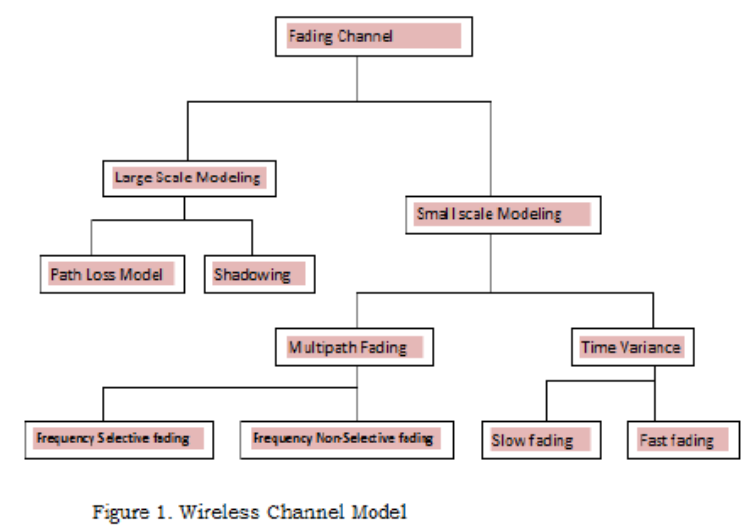

Basically propagation models are of two types:

1 Plane earth propagation. 2. Free space propagation

\subsection{Plane Earth Propagation Model:}

The affects of propagation model on ground is not considered for the free space propagation model. Some of the power will be reflected due to the presence of ground and then received by the receiver when a radio wave propagates over ground. The free space propagation model is modified and referred to as the „PlainEarth" propagation model by determining the effect of the reflected power. Thus this model suits better for the true characteristics of radio wave propagation over ground. This model computes the received signal to be the sum of a direct signal which reflected from a smooth, flat earth. The relevant input parameters include, the length of the path, the antenna heights, the operating frequency and the reflection coefficient of the earth. The coefficient will vary according to the type of terrain either water, wet ground, desert etc. The plane earth model in not appropriate for mobile GSM systems as it does not consider the reflections from buildings, multiple propagation or diffraction effects. Furthermore, if the mobile height changes (as it will in practice) then the predicted path loss will also be changed.

\section{ii. Propagation over a Plane Earth}

If we consider the effect of the earth surface, the main effect is that signals reflected off the earth surface may (partially) cancel the line of sight wave.

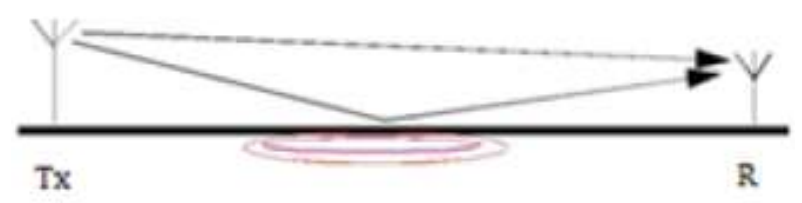

Figure 2. Propagating over plane earth

Table.1 for different heights of transmitter and receiver with fixed carrier frequency and Distance $b / w \mathbf{w x}$ and $\mathrm{Rx}=1 \mathrm{~km}$ :

\begin{tabular}{|l|l|l|}
\hline parameter & $\begin{array}{l}\text { At carrier frequency } 100 \mathrm{Mhz} ; \\
\text { Transmitter Height }=30 \mathrm{mts} ; \\
\text { Receiver Height }=30 \mathrm{mts} ; \\
\left(\epsilon_{r}\right)=15\end{array}$ & $\begin{array}{l}\text { At carrier frequency } 100 \mathrm{Mhz} ; \\
\text { Transmitter Height }=10 \mathrm{mts} ; \\
\text { Receiver Height }=50 \mathrm{mts} ; \\
\left(\epsilon_{r}\right)=15\end{array}$ \\
\hline $\begin{array}{l}\text { Horizontal } \\
\text { polarization(R) }\end{array}$ & 0.9685 degrees & 0.9788 degrees \\
\hline $\begin{array}{l}\text { Vertical } \\
\text { Polarization }(\mathrm{R})\end{array}$ & 0.6127 degrees & 0.7237 degrees \\
\hline
\end{tabular}

\subsection{Free Space Propagation}

The free space propagation model assumes a transmit antenna and a receive antenna to be located in an otherwise empty environment. Neither absorbing obstacles nor reflecting surfaces are considered. In particular, the influence of earth surface is assumed to be entirely absent. 


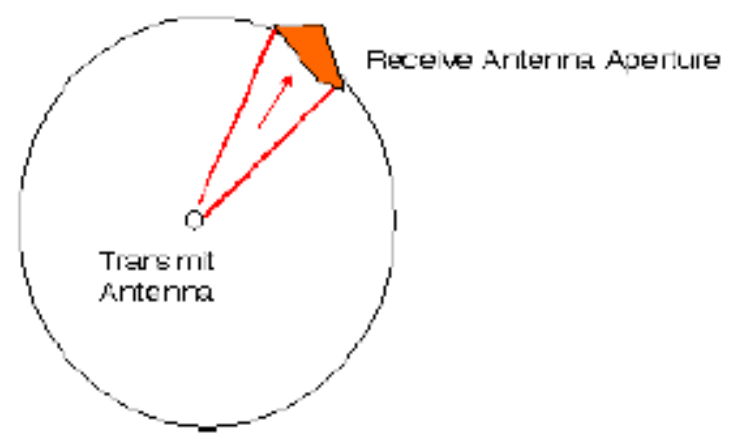

Figure 3 : Transmit antenna modeled as a point source Transmit power is spread over the surface area of a hypothetical sphere. The receiver antenna has an aperture $A$, illustrated in orange The surface area of a sphere of radius $d$ is $4 \pi d^{2}$. The power density $w$ at distance $d$ from a transmitter with power $p_{T}$ and antenna gain $G$, is

$$
w=p_{T} G_{t} /\left(4 \pi d^{2}\right)
$$

The available power $p_{R}$ at a receive antenna with gain $G_{R}$ is

$$
p_{R}=\frac{p_{T} G_{T}}{4 \pi d^{2}} \cdot A=\frac{\lambda^{2}}{(4 \pi d)^{2}} G_{T} p_{T} G_{R}
$$

where $A$ is the effective area or 'aperture' of the antenna, with $G_{R}=4 \pi A / \lambda^{2}$. The wavelength is $c / f_{c}$ with $c$ the velocity of light and $f_{c}$ the carrier frequency. The product $G_{t} p_{T}$ is called the effectively radiated power (ERP) of the transmitter.

\section{Path Loss Law}

As the propagation distance increases, the radiated energy is spread over the surface of a sphere of radius $d$, so the power received decreases proportional to $d^{2}$. Expressed in $\mathrm{dB}$, the received power is

$$
P_{d B}=P_{o}-20 \log d / d_{o}
$$

the path loss $L$ between two isotropic antennas $\left(G_{R}=1, G_{t}=1\right)$ can be expressed as $L=-\mathbf{3 2 . 4 4}-20 \log f_{c}-20 \log d$, where the loss is found in $\mathrm{dB}$

Table 2. path loss vs carrier Frequency for standard distance $1 \mathrm{Km}$

\begin{tabular}{|l|l|l|l|l|l|l|}
\hline Carrier frequency (Mhz) & 10 & 60 & 100 & 400 & 600 & 900 \\
\hline Distance (metres) & 1000 & 1000 & 1000 & 1000 & 1000 & 1000 \\
\hline Attenuation (in dB) & 52.44 & 68.00 & 72.44 & 84.48 & 88.00 & 91.53 \\
\hline
\end{tabular}

Table3. Path loss vs Carrier frequency for $5000 \mathrm{~km}$

\begin{tabular}{|l|l|l|l|l|l|l|}
\hline Carrier frequency(MHz) & 10 & 60 & 100 & 400 & 600 & 900 \\
\hline Distance(meters) & 5000 & 5000 & 5000 & 5000 & 5000 & 5000 \\
\hline Attenuation (in dB) & 66.42 & 81.98 & 86.42 & 98.46 & 101.98 & 105.50 \\
\hline
\end{tabular}

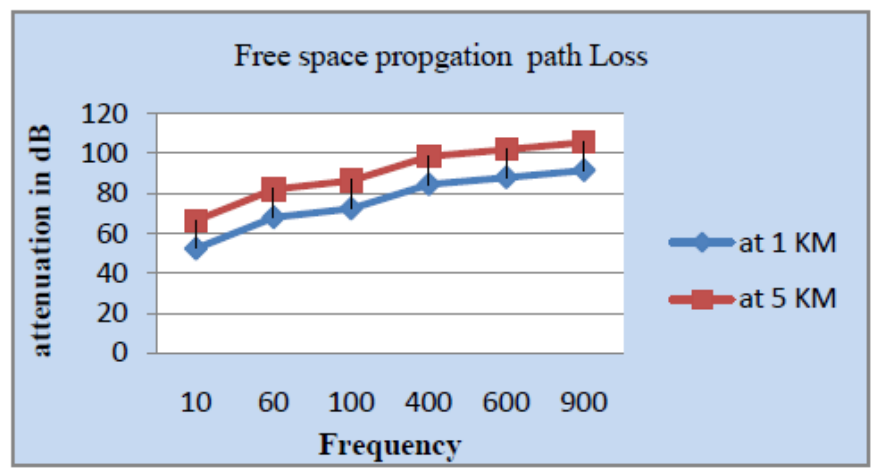

Figure 4.Uniform free space loss at different ranges of transmitter and receiver 


\section{iii. Egli's Model}

In contrast to the theoretical plane earth loss, Egli measured a significant increase of the path loss with the carrier frequency $f_{c}$ for ranges $1<d<50 \mathrm{~km}$. He proposed the semi-empirical model.

Table4. Losses present for various ranges between $\mathrm{Tx}$ and $\mathrm{Rx}$

\begin{tabular}{|c|c|c|c|c|c|}
\hline $\begin{array}{l}\text { Distance } \\
\text { (Meters) }\end{array}$ & 50 & 300 & 1000 & 2000 & 3000 \\
\hline $\begin{array}{l}\text { Attenuation in } \\
\mathrm{dB}\end{array}$ & $\begin{array}{l}\text { Not valid } \\
\text { distance } \\
\text {.It is free } \\
\text { space loss }\end{array}$ & $\begin{array}{l}\text { Not valid } \\
\text { distance } \\
\text { It is a free } \\
\text { space loss }\end{array}$ & $\begin{array}{l}\text { Not valid } \\
\text { distance } \\
\text { It is a free } \\
\text { space loss }\end{array}$ & $\begin{array}{l}84.43 \\
\text { Valid distance. } \\
\text { Egli path loss }\end{array}$ & $\begin{array}{l}91.48 \\
\text { Valid } \\
\text { distance. } \\
\text { Egli path loss }\end{array}$ \\
\hline
\end{tabular}

Egli's has proposed the path loss must be less than theoretical free space loss i.e., he introduced a frequency dependent empirical correction $\left(40 \mathrm{MHz} / f_{c}\right)^{2}$ for carrier frequencies $30 \mathrm{MHz}<f_{c}<1 \mathrm{GHz}$. To achieve the condition adjust heights of the Transmitter and receiver as required

$$
p_{R}=\left(\frac{40 M H z}{f_{c}}\right)^{2} \frac{\left(h_{T} h_{R}\right)^{2}}{d^{4}} p_{T} G_{T} G_{R}
$$

Table 5 : Fixed heights but different carrier frequencies:

\begin{tabular}{|l|l|l|l|l|}
\hline parameter & \multicolumn{2}{|l|}{$\begin{array}{l}\text { Carrier Frequency=100MHz; } \\
\text { Transmitter height=20 mts; } \\
\text { Receiver height }=20 \mathrm{metre}\end{array}$} & $\begin{array}{l}\text { Carrier Frequency }=900 \mathrm{MHz} \\
\text { Transmitter height=20 mts; } \\
\text { Receiver height =20metre }\end{array}$ \\
\hline Distance (Meters) & Up to 699 & 700 & Up to 670 & 671 \\
\hline Attenuation in dB & $\begin{array}{l}\text { Not valid distance } \\
\text { Only free space loss }\end{array}$ & 69.72 & $\begin{array}{l}\text { Not valid distance } \\
\text { Only free space loss }\end{array}$ & 88.07 \\
\hline
\end{tabular}

\section{Diffraction Loss:}

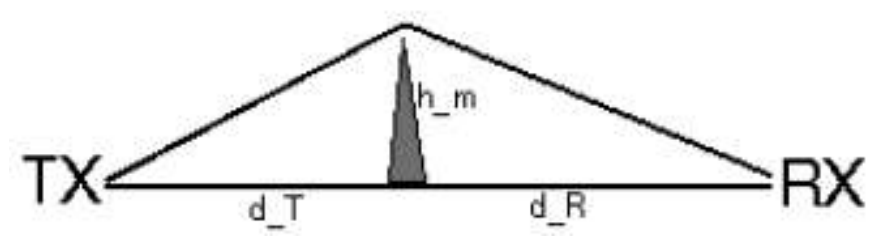

Figure 5: Path profile model for (single) knife edge diffraction

If the direct line-of-sight is obstructed by a single knife-edge type of obstacle, with height $h_{m}$ we define the following diffraction parameter $v$ :

$$
\nu=n_{\pi}\left(\sqrt{\frac{2}{\lambda}\left(\frac{1}{d_{T}}+\frac{1}{d_{R}}\right)}\right.
$$

where $d_{t}$ and $d_{R}$ are the terminal distances from the knife edge. The heights must be out of the field of antennas The diffraction loss, additional to free space loss and expressed in $\mathrm{dB}$, can be approximated by

\begin{tabular}{|ll|}
\hline$A_{D}=0$ & if $v<0$ \\
$A_{D}=6+9 v+1.27 v^{2}$ & if $0<v<2.4$ \\
$\Delta_{n}=12+70 \ln \sigma v$ & if $v>94$ \\
\hline
\end{tabular}


Table 6: different cases of diffraction losses:

\begin{tabular}{|l|l|}
\hline $\begin{array}{l}\text { a) distance between receiver and obstacle }=80 \mathrm{mts} \\
\text { b) distance between transmitter and obstacle }=90 \mathrm{mts} \\
\text { c) height of the obstacle }=5 \mathrm{metre} \\
\text { d) carrier frequency }=100 \mathrm{Mhz}\end{array}$ & $\begin{array}{l}\text { SKE loss }=57.05 \mathrm{~dB} \\
\text { Free space loss }=11.14 \mathrm{~dB} \\
\text { Over all attenuation }= \\
68.19 \mathrm{db}\end{array}$ \\
\hline $\begin{array}{l}\text { a) distance between receiver and obstacle }=100 \mathrm{mts} \\
\text { b) distance between transmitter and obstacle }=90 \mathrm{mts} \\
\text { c) height of the obstacle }=5 \mathrm{metre} \\
\text { d) carrier frequency }=100 \mathrm{Mhz}\end{array}$ & $\begin{array}{l}\text { SKE loss }=58.01 \mathrm{~dB} \\
\text { Free space loss }=10.89 \mathrm{~dB} \\
\text { Over all attenuation } \\
68.90 \mathrm{db}\end{array}$ \\
\hline $\begin{array}{l}\text { a) distance between receiver and obstacle }=60 \mathrm{mts} \\
\text { b) distance between transmitter and obstacle }=90 \mathrm{mts} \\
\text { c) height of the obstacle }=5 \mathrm{metre} \\
\text { d) carrier frequency }=100 \mathrm{Mhz}\end{array}$ & $\begin{array}{l}\text { SKE loss }=55.96 \mathrm{~dB} \\
\text { Free space loss }=11.53 \mathrm{~dB} \\
\text { Over all attenuation } \\
67.49 \mathrm{db}\end{array}$ \\
\hline $\begin{array}{l}\text { a) distance between receiver and obstacle }=60 \mathrm{mts} \\
\text { b) distance between transmitter and obstacle }=80 \mathrm{mts} \\
\text { c) height of the obstacle }=5 \mathrm{metre} \\
\text { d) carrier frequency }=100 \mathrm{Mhz}\end{array}$ & $\begin{array}{l}\text { SKE loss }=55.36 \mathrm{~dB} \\
\text { Free space loss }=11.65 \mathrm{~dB} \\
\text { Over all attenuation }= \\
67.01 \mathrm{db}\end{array}$ \\
\hline
\end{tabular}

Many measurements of propagation losses for paths with combined diffraction and ground reflection losses indicate that knife edge type of obstacles significantly reduce ground wave losses. Blomquist suggested two methods to find the total loss and the empirical formula

Many measurements of propagation losses for paths with combined diffraction and ground reflection losses indicate that knife edge type of obstacles significantly reduce ground wave losses. Blomquist suggested two methods to find the total loss and the empirical formula

$$
A_{B_{2}}=A_{f_{x}}+\sqrt{A_{r}^{2}+A_{d}^{2}} \quad A_{B_{1}}=A_{f i}+A_{r}+A_{d}
$$

where $A_{f s}$ the free space loss, $A_{R}$ the ground reflection loss and $A_{D}$ the multiple knife-edge diffraction loss in $\mathrm{dB}$ values.

Many measurements of propagation losses for paths with combined diffraction and ground reflection losses indicate that knife edge type of obstacles significantly reduce ground wave losses. Blomquist suggested two methods to find the total loss and the empirical formula where $A f s$ the free space loss, $A R$ the ground reflection loss and $A D$ the multiple knife-edge diffraction loss in $\mathrm{dB}$ values.

\section{Empirical Propagation Models}

Okumura and hata are among the two empirical propagation models. The two basic propagation models are free space loss and plane earth loss would be requiring detailed knowledge of the location and constitutive parameters of building, terrain feature, every tree and terrain feature in the area to be covered. It is too complex to be practical and

would be providing an unnecessary amount of detail therefore appropriate way of accounting for these complex effects is by an empirical model. There are many empirical prediction models like,

- $\quad$ EGLI's model

- OKUMURA's model

- HATA's model

- $\quad$ COST 231 - HATA

- $\quad$ SAKAGAMI- KUBOI model,

- BERTONI-WALFISCH MODEL

- $\quad$ IKEGAMI model

\subsection{Channel Models:}

A macrocell is a cell in a mobile phone network that provides radio coverage served by a high power cellular base station (tower). Generally, macrocells provide coverage larger than micro cell. The antennas for macro cells are mounted on ground-based masts, rooftops and other existing structures, at a height that provides a clear view over the surrounding buildings and terrain. Macrocell base stations have power outputs of typically tens of watts. Macrocell performance can be increased by increasing the efficiency of the transreciever. The term macrocell is used to describe the widest range of cell sizes. Macrocells are found in rural areas or along highways. 


\section{Micro-Cellular Path Loss}

Microcellular networks use a cell size of, say, 200 to 2,000 meters. Propagation models for micro-cellular communication typically model the path loss law as a transition from free space propagation to ground wave propagation . if $d<d_{g}$ where theoretically the turnover distance $d_{g}$ occurs at $d_{g}<4 h_{R} h_{T} /$, where $d$ is the distance of the radio link under study, $h_{R}$ and $h_{T}$ are the heights of the receiving and transmitting antenna respectively, and $\lambda$ is the wavelength of the transmitted wave.

\section{Indoor Wireless RF Channels}

The vehicular cellular phone systems initiated a rapid growth of wireless communication. However, with the growth of these systems cell sizes are made smaller and smaller to increase user capacity. Examples of indoor systems are telephony (cardless phones and wireless PABX-es) and data services (e.g. wireless LAN"es). The indoor channel can less easily be captured in rough path loss exponents. While delay spreads are often much smaller than outdoors, the indoor systems often have to carry very high data rates, e.g. to support wireless multimedia computing. There are several causes of signal corruption in a wireless channel. The primary causes of attenuation are distance, penetration losses through walls and floors and multipath propagation.

These models can be broadly categorized into three types; empirical, deterministic and stochastic. Empirical models are those based on observations and measurements alone. These models are mainly used to predict the path loss, but models that predict rain-fade and multipath have also been proposed. The deterministic models make use of the laws governing electromagnetic wave propagation to determine the received signal power at a particular location. Deterministic models often require a complete 3-D map of the propagation environment

\subsection{Path Loss and Coverage Prediction i.Deterministic approach}

Ray tracing allows deterministic prediction of signal level received at various indoor locations. In ray tracing, a large collection of possible propagation paths is evaluated and the amplitude and delay of each relevant path is considered. For narrowband coverage prediction an accuracy of about $2 \mathrm{~dB}$ has been achieved, but this requires a high-resolution 3D data base of the environment, accurate knowledge of building materials and calibration of

predictions against actual measurements.

\section{ii. Statistical approach}

Signal attenuation over distance is observed when the mean received signal power is attenuated as a function of the distance. For indoor propagation the mechanism effects a wave guidance through corridors can occur. The path loss typically is of the form

\section{Power $=$ distance}

The path loss exponent $n$ may range from about 2 (in corridors) to 6 (for cluttered and obstructed paths). For frequencies between $800 \mathrm{MHz}$ and $1.9 \mathrm{GHz}$, COST 231 reports the following values for the path loss exponent $n$ :

Table. 7 Range of exponent $\mathrm{n}$ for different environments

\begin{tabular}{|l|l|l|}
\hline Environment & Exponent $\mathbf{n}$ & $\begin{array}{l}\text { Propagation } \\
\text { Mechanism }\end{array}$ \\
\hline Corridors & $1.4-1.9$ & Wave guidance \\
\hline Large open & 2 & Free space loss \\
\hline Furnished & 3 & Free space Loss + multipath \\
\hline Densely & 4 & Non-LOS, diffraction, scattering \\
\hline Between & 5 & Losses during floor / wall \\
\hline
\end{tabular}

Other models predict that the indoor path loss follows the law:

$$
L=L_{F S}+c \text { distance }
$$

where $c$ is on the order of 0.2 to $0.6 \mathrm{~dB}$ per meter.This models has been proposed for metropolitan office buildings, for propagation distances from 1 to 100 meter and frequencies between $900 \mathrm{MHz}$ and $4 \mathrm{GHz}$. 


\section{b.Multipath}

The results of multipath are from the fact that the propagation channel consists of several obstacles and reflectors.Thus, the received signal arrives as an unpredictable set of reflections and / or direct waves each with its own degree of attenuation and delay. In indoor multipath waves tend to arrive in clusters. Within one cluster, paths have relatively small differences in delay. Delays between clusters are larger.

\section{Rate Of Fading}

Time variation of the channel occurs if the communicating device (antenna) and components of its environment are in motion. Fortunately, the degree of time variation within an indoor system is much less than that of an outdoor mobile system. For wireless LAN"s this could mean that an antenna place in a local multipath null, remains in fade for a very long time. Measures such as diversity are needed to guarantee reliable communication irrespective of the position of the antenna. Wideband transmission could provide frequency diversity.

\section{Path Loss, Wall Penetration And Cell Layout}

An important issue for indoor cellular reuse systems is the possibility of interference from users in adjacent cells. In designing cells it would be convenient if natural barriers such as walls and ceilings/floors could be used as cell boundaries.

\begin{tabular}{|l|c|c|}
\hline $\begin{array}{l}\text { Attenuation } \\
\text { Factor }\end{array}$ & $900 \mathrm{MHz}$ & $1700 \mathrm{MHz}$ \\
\hline Floor & $10 \mathrm{~dB}$ & $16 \mathrm{~dB}$ \\
\hline
\end{tabular}

A signal at $1.2 \mathrm{GHz}$ traversing a wall looses 3 to $8 \mathrm{~dB}$ of its energy. User experience with wireless LANs is that in the 2.4 and $5 \mathrm{GHz}$ bands, communications signal propagate through a limited number walls and ceilings, but at higher frequencies $(17 \mathrm{GHz})$ the signal is very weak after attenuation by a concrete or brick wall. An appropriate statistical model can be to assume a building penetration loss of $12 \mathrm{~dB}$ with a standard deviation of $10 \mathrm{~dB}$.

\subsection{Okumura Propagation Model}

Okumura"s model was developed during the mid 1960's as the result of large-scale propagation model is one of the most frequently used macroscopic propagation models. conducted in and around Tokyo. The model was designed for use in the frequency range 150 up to $2000 \mathrm{MHz}$ and mostly in an urban propagation environment. Formula for Okumura Model is expressed below .Okumura"s model assumes that the path loss between the TX and RX in the terrestrial propagation environment can be expressed as:

$$
L_{\text {Medinu }}=L_{F S}+A_{m u}+H_{t u}+H_{n u}
$$

$\begin{array}{ccl}\text { where: } & L_{\mathrm{Median}}{ }^{-} & \text {Median path loss between the TX and RX expressed in dB } \\ & L_{F S^{-}} & \text {Path loss of the free space in } \mathrm{dB} \\ A_{m u^{-}} & \text {"Basic median attenuation" -additional losses due to propagation }\end{array}$

in Urban environment in $\mathrm{dB}$

$$
\begin{aligned}
& H_{t u} \text { - TX height gain correction factor in } \mathrm{dB} \\
& H_{x u}-\mathrm{RX} \text { height gain correction factor in } \mathrm{dB}
\end{aligned}
$$

The free space loss term can be calculated analytically using

$$
\mathrm{L}_{\mathrm{FS}}=32.45+20 \log (\mathrm{d})+20 \log (\mathrm{f})-10 \log \left(\mathrm{G}_{\mathrm{T}}\right)-10 \log \left(\mathrm{G}_{\mathrm{R}}\right)
$$

Where $d$ is the distance between transmitter and receiver in Kilo meters

$f$ is operating frequency in Mega hertz

$\mathrm{G}_{\mathrm{T}}$ transmitting antenna gain

$\mathrm{G}_{\mathrm{R}}$ is receiving antenna gain

The effective antenna height is calculated as the height of the antenna"s radiation above the average terrain. The terrain is averaged along the direction of radio path over the distances between three and fifteen kilometers .Due to its simplicity and the fact that it is one of the first models developed for the mobile cellular propagation environment, Okumura"s model is one of the most widely used models. Some difficulties are:

1. If the average height of the terrain is above the height of the radiation centerline, the effective antenna

2. height may become negative.

3. The whole empirical nature of the Okumura model means that its applicability is limited to parameter

4. ranges used in the model development 
5. Use of the effective antenna height is limited to the cases of large cell radii. If the cell radius is smaller

6. than $3 \mathrm{~km}$, the use of effective antenna height does not seem appropriate.

\subsection{Hata Model:}

Hata established empirical mathematical relationships to describe the graphical information given by Okumura. Hata ${ }^{e e}$ formulation is limited to certain ranges of input parameters and is applicable only over quasismooth terrain.

The mathematical expression and their ranges of applicability are as follows

Carrier Frequency: $150 \mathrm{MHz} \leq \mathrm{f}_{\mathrm{c}} \leq 1500 \mathrm{MHz}$

Base Station (BS) Antenna Height: $30 \mathrm{~m} \leq \mathrm{h}_{\mathrm{b}} \leq 200 \mathrm{~m}$

Mobile Station (MS) Antenna Height: $1 \mathrm{~m} \leq \mathrm{h}_{\mathrm{m}} \leq 10 \mathrm{~m}$

\section{Transmission Distance: $\mathbf{1} \mathbf{~ k m} \leq \mathbf{d} \leq \mathbf{2 0} \mathbf{~ k m}$}

\begin{tabular}{|ll}
\hline$A+B \log _{10}(d)$ & for urban areas \\
$L_{p}(d B)=A+B \log _{10}(d)-C$ & for suburban area \\
$A+B \log _{10}(d)-D$ & for open area
\end{tabular}

\section{Where}

$$
\begin{aligned}
& A=69.55+26.16 \log _{10}\left(f_{c}\right)-13.82 \log _{10}\left(h_{b}\right)-a\left(h_{m}\right) \\
& B=44.9-6.55 \log _{10}\left(h_{b}\right) \\
& C=5.4+2\left[\log _{10}\left(f_{c} / 28\right)\right]^{2} \\
& D=40.94+4.78\left[\log _{10}\left(f_{s}\right)\right]^{2}-18.33 \log _{10}\left(f_{s)}\right.
\end{aligned}
$$

Where, $\mathbf{a}\left(\mathbf{h}_{\mathrm{m}}\right)=$

$$
\begin{array}{ll}
{\left[1.1 \log _{10}\left(f_{c}\right)-0.7\right] h_{m}-\left[1.56 \log _{10}\left(f_{c}\right)-0.8\right] \text { for medium or small cities }} \\
8.29\left[\log _{10}\left(1.54 h_{m}\right)\right]^{2}-1.1 & \text { for large city and } f_{c} \leq 200 \mathrm{MHz} \\
3.2\left[\log _{10}\left(11.75 h_{m}\right)\right]^{2}-4.97 & \text { for large city and } f_{c} \geq 400 \mathrm{MHz}
\end{array}
$$

\subsection{COST-231 Model}

The COST231-Hata model extends Hata's model for use in the $1500-2000 \mathrm{MHz}$ frequency range, where it is known to underestimate path loss. The model is expressed in terms of the following parameters Most future PCS systems are expected to operate in the $1800-2000 \mathrm{MHz}$ frequency band.

The path loss according to the COST-231-Hata model is expressed as:

$$
L_{p}(d B)=A+B \log 10(d)+C
$$

$$
\begin{aligned}
& \text { Where; } \quad \begin{array}{l}
A=46.3+33.9 \log 10\left(f_{c}\right)-13.28 \log 10\left(h_{b}\right)-a\left(h_{m}\right) \\
B=44.9-6.55 \log 10\left(h_{b}\right) \\
C=\quad 0 \quad \text { for medium city and suburban areas } \\
3 \quad \text { for metropolitan areas }
\end{array} \\
& \begin{array}{l}
\text { C } \\
\quad
\end{array}
\end{aligned}
$$

The common representation formula of different communication models is

$P L(d)=P L(d 0)+10$ nlog10

\section{Where}

$\mathrm{d}=$ Distance between Transmitter station and Mobile station

$d_{o}=$ Reference point

$\mathrm{n}=$ Path loss exponent 
Table 8 .Comparison of path loss of communication models with respect to distance

\begin{tabular}{|c|c|c|c|}
\hline Distance(km) & Olaumura path loss(db) & Hata path loss(db) & Cost-231path loss(db) \\
\hline 0 & 20 & 200 & 250 \\
\hline 2 & 57 & 250 & 298 \\
\hline 8 & 62 & 258 & 302 \\
\hline 12 & 71 & 262 & 310 \\
\hline 16 & 72.2 & 270 & 315 \\
\hline 18 & 72.3 & 272 & 317 \\
\hline
\end{tabular}

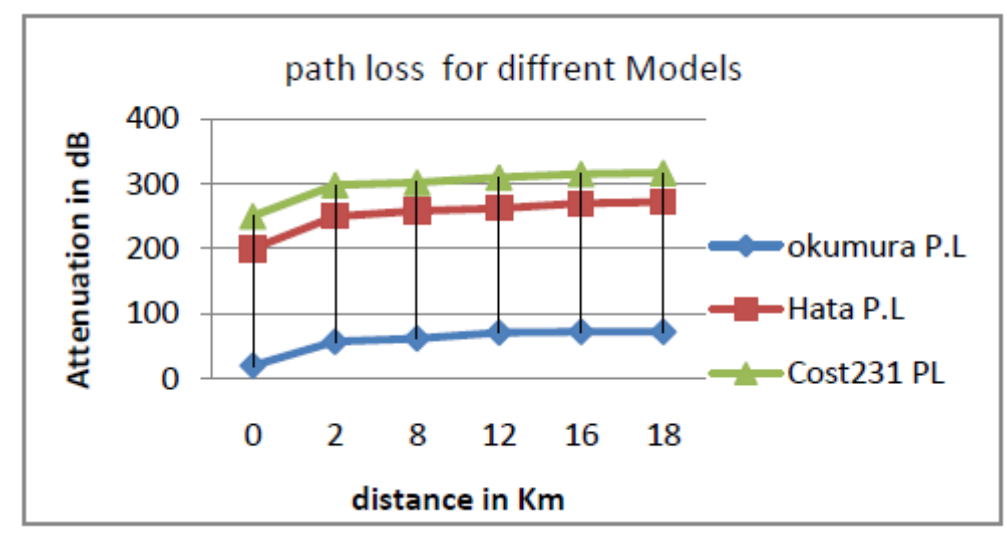

Figure 6. Path Loss variations for Different propagation Models:

\subsection{Bertoni-Walfisch Model}

The model of BERTONI-WALFISCH takes into account positioning of buildings 1 , influence on a communication mobile radio. He assumes that spread is made in most cases by diffraction at the top of buildings being in the neighborhood of the mobile receiver. It considers that attenuation of course am composed of three parties:

- Attenuation between two antennae in free space

- Attenuation sudden by the field at the top of building, who is owed to the losses of diffraction across a

- $\quad$ series of rows building.

- The losses of diffraction at the top of building neighbor of the motive.

The total attenuation is expressed as follows:

$$
A f=A f 0+A f 1
$$

With: $A f 0$ : is the attenuation in free space given by relation

$$
A f 0=32.4+20 \log (f)+20 \log (D)
$$

Af1: correction term which takes into account the curvature of the earth and the urban environment.

$$
A f 1=57.1+\log (f)+A-18 \log \left(h_{b}\right)+18 \log (D)-18 \log \left(1-\mathbf{D}^{2} / 17 \mathbf{h}_{s}\right)
$$

$$
A=5 \log \left[(\mathbf{d} / 2)^{2}+\left(h_{b}-h_{m}\right)^{2}\right)-9 \log (d)+20 \log \left(\operatorname{Tan}-1\left(2\left(h_{d}-h_{b} / d\right)\right]\right.
$$

Where: $D$ : Distance in $\mathrm{Km}$.

$$
\begin{aligned}
& f: \text { Frequency in MHz. } \\
& d \text { : Distance between buildings in (m). } \\
& h_{b} \text { : The medium height of buildings in (m). } \\
& h s: \text { The height of the basic station. } \\
& h_{m}: \text { Height of the motive in }(\mathrm{m}) .
\end{aligned}
$$

This model is applicable to urban areas and suburban. It assumes that the antenna heights of base stations are quite high and surrounded by rows of buildings of similar height and regularly spaced apart by a distance $\mathrm{d}$. 


\subsection{IKEGAMI Model}

It is based on the theory of geometric perspective, where they consider the spread of the wave restricted in two rays. He assumes moreover, an ideal structure of a city with an uniform height of buildings. It is expressed by following relation:

$$
A f=A f 0+A f 1
$$

With: $A f 0$ : Free-space loss given previously (model of BERTONI-WALFISCH).

Af1: Weakening of reflection, diffraction, it is given by:

$$
A f 1=-5.8-10 \log \left(1+3 / L^{2}\right)-\log (w)+20 \log \left(h_{b}-h_{m}\right)+20 \log (\sin \phi)+10 \log (f)
$$

With: $\phi$ : Orientation of the street in comparison with the incidental ray (in degree)

\section{$\mathbf{h}_{b}$ : Medium height of buildings.}

L: The coefficient of cogitation of buildings is. Ikegami assumes that buildings introduce weakening of $6 \mathrm{~dB}$.

\subsection{Sakagami-Kuboi Model}

This analysis is based on measurements performed in the Japan in urban circles. These measurements are analyzed by the procedure of numerous declines to find the influence of parameters characterizing urban middle on the weakening of Spread
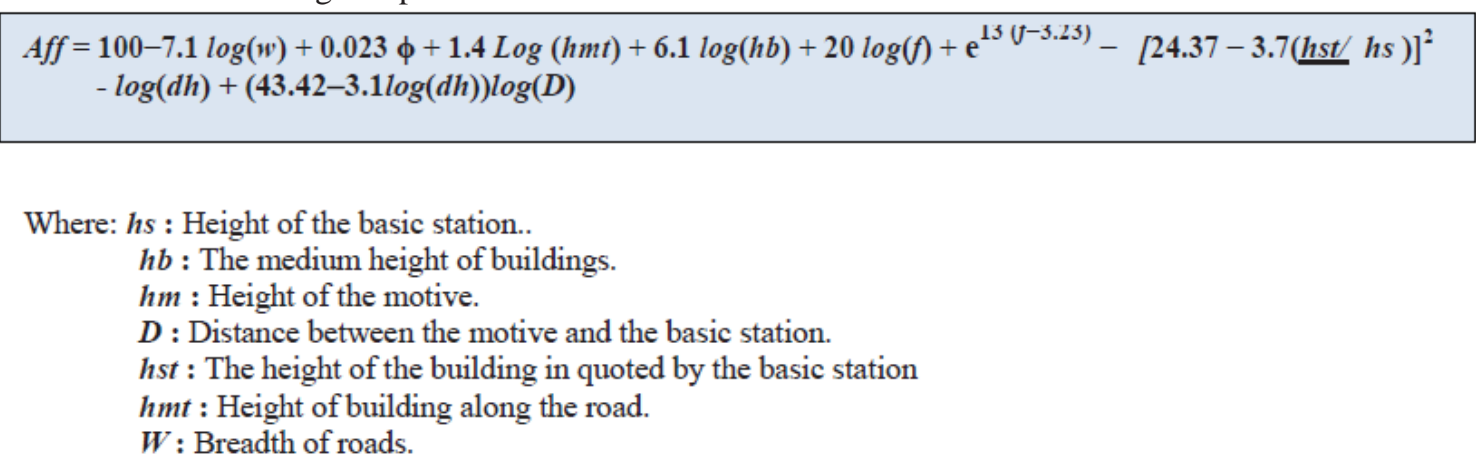

\section{Results}

The frees pace path loss for $1 \mathrm{~km}$ and $5 \mathrm{~km}$ distances of transmitter and receiver are observed as they are uniform For different heights of transmitter and receiver with fixed carrier frequency at $1 \mathrm{~km}$ Distancebetween Transmitting and receiving antennas is tabulated in Table 1.also found path Losses present for various ranges between Transmitter and Receiver at Fixed heights but different carrier frequencies are shown in Table 5.observed the various diffraction losses trend with respect to distance between receiver which are shown in Table 6. Path Loss variations for Different propagation Models are shown in Figure 6.Thsi paper also includes many empirical prediction models like Okumura and hata etc.and concluded Okumura model shows the least path loss and Cost-231 model shows the largest path loss are Shown in Table 8.Finally This paper motive is to produce detailed knowledge about all wireless propagation models and empirical models of channel

\section{References}

[1]. T. K. Sarkar, Z. Ji, K. Kim, A. Medour \& M. S.Palma, "A Survey of Various Propagation Models for Mobile Communication", IEEE Antennas and Propagation Magazine, Vol. 45, No. 3, June 2003

[2]. Zia Nadir, Member, IAENG, Muhammad Idrees Ahmad, "pathloss determination using Okumura-hata model and cubic regression for missing data for Oman" IMECS 2010 ,March 17-19

[3]. Z. Nadir \& M. Idrees Ahmad, "Path loss Determination Using Okumura-Hata Model and Cubic Regression for Missing Data for Oman", Proceeding of IMECS, Vol. 2,2010.

[4]. H. K. Sharma, S. H. Sahu \& S. Sharma, "Enhanced cost 231 propagations model in wireless network"International journal of computer application (0975 - 8887) Vol. 19, No. 06, April 2011.

[5]. Z. Nadir, N. Elfadhil, F. Touati, "Pathloss determination using Okumura-Hata model and spline interpolation for missing data for Oman" World Congress on Engineering, IAENG-WCE-2008, Imperial College, London, United Kingdom, 2-4 July,2008. 
[6]. S. sarooshyari \& N. Madaya, "An Introduction to mobile radio propagation and characterization of frequency bands" wireless comm. Technologies, IEEE, 16:332:559,1996.

[7]. Wireless communications principles and practice second edition by Theodore S.Rappaport

[8]. P. K. Sharma \& R. K. Singh, "Comparative Analysis of Propagation Path loss Models with Field Measured Data", International Journal of Engineering Science and Technology Vol. 2(6), 2010, pp 2008-2013.

[9]. V. S. Abhayawardhana, I. J. Wassell, D. Crosby, M. P. Sellars \& M. G. Brown,” Comparison of Empirical Propagation Path Loss Models for Fixed Wireless Access Systems", IEEE, December 2003.

[10]. M. Kumar, V. Kumar \& S. Malik, "Performance and analysis of propagation models for predicting RSS for efficient handoff", International journal of advanced scientific research \& technology, Vol.1, Issue 2, February 2012.

[11]. N. L. B. M. Nordon, "Interface developing for Hata model using Matlab”, Universiti Teknologi Malaysia, May 2008.

[12]. A. katariya, A. yadav, N. Jain \& G. tomar, "BER performance criteria based on standard IEEE802.11a forOFDM in multipath fading environment", International Conference on Computational Intelligence and Communication Systems, 2011.

[13]. Spectrum Planning Team, "Investigation of Modified Hata Propagation Models", IEEE, April 2001.

[14]. M. A. Masud, M. Samsuzzaman \& M. A. Rahman, "Bit Error Rate Performance Analysis on ModulationTechniques of Wideband Code Division Multiple Access", Journal Of Telecommunication, Volume 1, Issue 2, PP.22-29, March 2010.

[15]. A. A. Tahir \& F. Zhao, "Performance analysis on modulation techniques of W-CDMA in multipath fading channel ", January 2009.

[16]. O. Grigoriadis \& H. Srikanth Kamath, "Ber Calculation Using Matlab Simulation For OFDM Transmission”, IMECS, Vol.2, 2008.

[17]. C. Akkash, "Methods for Path loss Prediction", Report 09067, ISSN 1650-2647, Oct. 2009.

[18]. H. Cavdar," A Statistical Approach to Bertoni - Walfisch Propagation Model for Mobile Radio Design in Urban area, IEEE, PP. 279-283, 2001.

[19]. M. Dottling, A. Jahn \& W. Wiesbeck, “A comparison and verification of 2D and 3D ray tracing propagation models for land mobile satellite communications", IEEE, 0-7803-6369, PP. 434-437, 2000.

[20]. Crane, R. K. (1980). Prediction of attenuation by rain, IEEE Transactions on communications, COM-28, p.1727-1732, September. 